\title{
As atribuições do fisioterapeuta do Núcleo Ampliado a Saúde da Família e Atenção Básica no município de Lucena-PB
}

The attributions of physiotherapist of the Nucleus Extended To Family Health and Basic Attention in the city of Lucena-PB Las atribuciones del Núcleo Fisioterapéutico Extendido a la Salud Familiar y la Atención Básica en la ciudad de Lucena-PB

\author{
Luiz Paulo Vicente FREIRE ${ }^{1}$ \\ Weslley Barbosa SALES ${ }^{2}$ \\ Daniella de Souza BARBOSA ${ }^{3}$ \\ Jairo Domingos de MORAIS
}

Fisioterapeuta, Especialização em Fisioterapia Traumato-Ortopédica e Desportiva, Faculdade Maurício de Nassau( UNINASSAU) 58030-000 João Pessoa-PB, Brasil ${ }_{2}^{2}$ Graduando em Fisioterapia, Faculdade Maurício de Nassau( UNINASSAU) 58030-000 João Pessoa-PB, Brasil

${ }^{3}$ Professora Adjunta, DPS- CCM Departamento de Promoção da Saúde, Universidade Federal da Paraíba (UFPB) 58051-900 João Pessoa-PB, Brasil

${ }^{4}$ Professor Adjunto, ICS Instituto de Ciências da Saúde,Universidade da Integração Internacional da Lusofonia Afro-Brasileira (UNILAB) 62790-000 Redenção- CE, Brasil

\begin{abstract}
Resumo
Objetivo: analisar a atuação do fisioterapeuta no NASF-AB no município de Lucena-PB. Material e método: Trata-se de uma pesquisa de campo cuja coleta de dados foi feita pela aplicação de dois questionários a uma amostra - por conveniência - composta por cinco fisioterapeutas que atuavam no NASF- AB no município de Lucena-PB por no mínimo seis meses. Resultados: Foi identificado que 100\% dos profissionais cumprem a carga horária exigida pelos profissionais do NASF-AB da modalidade 1 e que $60 \%$ desenvolvem todas as atividades cabidas ao fisioterapeuta do NASF- AB no município como atendimento domiciliar, atendimento ambulatorial na unidade mista de saúde e atividades educativas em saúde promovidas nos equipamentos sociais do território (unidades básicas de saúde e escolas públicas). Conclusão: Os fisioterapeutas que atuam no NASF-AB 1 da referida cidade litorânea paraibana têm desenvolvido ações de saúde como atividades domiciliares e ambulatoriais, atividades em grupo, atividades educativas em equipe. O processo de trabalho dos fisioterapeutas que atuam no NASF-AB pode contribuir para transformar a prática profissional no nível de Atenção Básica, ampliando suas competências e habilidades na atuação interdisciplinar e desenvolvimento de ações de prevenção de doenças e promoção à saúde nos territórios.
\end{abstract}

Descritores: Atenção Primária à Saúde; Saúde Pública; Fisioterapia.

\section{Abstract}

Objective: to analyze the Physiotherapist's performance in NASF-AB in the city of Lucena-PB. Material and method: This is a field research which data collection was done by applying two questionnaires to a sample - for convenience - composed of five physiotherapists who worked in NASF-AB in the city of Lucena-PB for at least six months. Results: It was identified that $100 \%$ of professionals fulfill time load required by NASF-AB professionals of modality 1 and that $60 \%$ develop all activities appropriate for the NASF-AB physiotherapist in the city as home care, ambulatory care in the mixed unit of health and educational activities in health promoted in the social facilities of the territory (basic health units and public schools). Conclusion: Physiotherapists working in the NASF-AB 1 of the coastal city of Paraiba have developed health actions such as home and outpatient activities, group activities, and educational activities in a team. Finally, it is necessary to verify how the work process of the physiotherapists working in the NASF-AB can contribute to transform the professional practice in the level of Basic Attention, extending its competences and abilities in the interdisciplinary action and development of disease prevention actions and health promotion in the territories.

Descriptors: Primary Health Care; Public Health; Physical Therapy Specialty.

\section{Resumen}

Objetivo: analizar el papel del fisioterapeuta en NASF-AB en la ciudad de Lucena-PB. material y método: Esta es una investigación de campo cuya recopilación de datos se realizó mediante la aplicación de dos cuestionarios a una muestra, por conveniencia, compuesta por cinco fisioterapeutas que trabajaron en NASF-AB en la ciudad de Lucena-PB durante al menos seis meses. Resultados e discussões: Se identificó que el $100 \%$ de los profesionales cumplen con la carga de trabajo requerida por los profesionales NASF-AB en la modalidad 1 y que el $60 \%$ desarrolla todas las actividades para el fisioterapeuta NASF-AB en la ciudad, como atención domiciliaria, atención ambulatoria en la unidad de atención mixta. actividades educativas de salud y salud promovidas en las instalaciones sociales del territorio (unidades básicas de salud y escuelas públicas). Conclusión: Los fisioterapeutas que trabajan en NASF-AB 1 en la referida ciudad costera de Paraíba han desarrollado acciones de salud como actividades domiciliarias y ambulatorias, actividades grupales, actividades educativas en equipo. El proceso de trabajo de los fisioterapeutas que trabajan en NASF-AB puede contribuir a transformar la práctica profesional a nivel de atención primaria, ampliando sus habilidades y capacidades en actividades interdisciplinarias y el desarrollo de acciones de prevención de enfermedades y promoción de la salud en los territorios.

Descriptores: Atención Primaria de Salud; Salud Pública; Fisioterapia.

\section{INTRODUÇÃO}

O primeiro contato do usuário nos serviços de atenção primária com o sistema de saúde visa atender as afecções mais frequentes e resolver a maioria dos problemas de saúde de uma população cuja as ações são vinculadas aos setores da Atenção Básica $(\mathrm{AB})$ e realizada por meio do trabalho em conjunto com a equipe multiprofissional nas Unidades Básicas de Saúde (UBS) e na Estratégia de Saúde da Família (ESF) ${ }^{1}$.

A estratégia de saúde da família vem reconhecendo e atuando sobe as condições de risco presente na comunidade, e buscando recursos coerentes com o contexto social, econômico e cultural em que essas comunidades se inserem. Nessa estratégia, a equipe de saúde, multiprofissional e interdisciplinar, é um importante dispositivo para reorganização do processo do trabalho ${ }^{2}$.

Considerando a consolidação da Estratégia Saúde da Família, a melhoria da qualidade e a reparabilidade, foi criado em 24 de janeiro de 2008, por meio da Portaria GM/MS n. 154, o Núcleo de Apoio à Saúde da Família (NASF), porém em 28 de Dezembro de 2012 foi republicada e passou a ser GM/MS n. 3.124 sendo esta sua atual portaria, contendo as modalidades do NASF 1,2 e 3. Em 2017, com a terceira edição da PNAB, o NASF passou a ser denominado de Núcleo Ampliado da Saúde da Família e Atenção Básica (NASF-AB), no qual tem 
como objetivo ampliar a abrangência e o sentido das ações de Atenção Básica, bem como sua eficácia e eficiência ${ }^{3}$.

O fisioterapeuta é um profissional que faz parte do NASF-AB e que sua inserção nos serviços de atenção básica à saúde é um processo em estruturação, que esteve associado durante algum tempo ao nascimento da profissão, quando este profissional era denominado como reabilitador, tratando apenas a doença e suas sequelas. Essa lógica durante muito tempo excluiu da rede básica os serviços de fisioterapia, acarretando uma grande dificuldade de acesso da população a esse serviço e impedindo o profissional de atuar na atenção básica ${ }^{4}$. Cabe ao fisioterapeuta segundo as diretrizes da ESF o desenvolvimento de ações e o provimento de serviços para tratamento e reabilitação, controle dos riscos e danos em seu território, prevenindo agravos e promovendo a saúde com ações de caráter individual, de grupos e da coletividade. O Fisioterapeuta deve atuar na Equipe Multiprofissional, através de uma abordagem interdisciplinar, objetivando a integralidade da assistência, e enfatizando aspectos de promoção, prevenção, reabilitação, explorando todo o arranjo ${ }^{5}$.

A atuação deve ocorrer, preferencialmente, no contexto coletivo, com o envolvimento e a participação da população. Esta atuação de forma integrada na equipe possui como objetivo: planejar, implementar, controlar e executar políticas, programas, cursos, pesquisas ou eventos de saúde pública. Possui um grande potencial regulador, podendo funcionar como uma ligação entre a comunidade e a equipe na elaboração das ações de saúde ${ }^{6}$.

Assim, o fisioterapeuta insere-se no sistema de atenção básica numa nova perspectiva de atuação gerencial, focada nas práticas de prevenção e promoção e não de uma forma exclusiva aos procedimentos de reabilitação, pelo contrário do que tem predominado nas ações deste segmento profissional $^{7}$. No fim dos anos 80 no qual ouve a implantação do Sistema Único de Saúde (SUS) no Brasil, inúmeras mudanças foram constatadas no campo da saúde, baseadas nos novos conceitos em relação aos saberes e práticas defendidos por estudiosos que acreditavam na atuação diferenciada dos profissionais frente ao processo saúde/doença ${ }^{8}$.

$\mathrm{O}$ conceito de promoção da saúde nos últimos anos tornou-se um marco para as políticas de saúde em nível mundial. O objetivo da promoção de saúde é reatar os laços existentes entre saúde e bemestar social, entre qualidade de vida coletiva e individual. No Brasil, o tema promoção da saúde ganhou força a partir das mudanças nas políticas de saúde, nos anos oitenta, que resultaram na Lei Orgânica da Saúde (1990) e na concepção do Sistema Único de Saúde?.
O surgimento da Fisioterapia no Brasil, não teve uma tradição ligada à Atenção Básica à Saúde pelo contrário, historicamente centralizou suas ações principalmente nos níveis de atenção secundário, referente ao diagnóstico e tratamento das doenças e terciaria responsável pela limitação dos danos e a reabilitação ${ }^{10}$.

A Fisioterapia, regulamentada pelo Decretolei n. 938/69, Lei n. 6.316/75, Resoluções do COFFITO, Decreto n. 9.640/84 e Lei n. 8.856/94, é definida como: uma ciência da saúde que estuda, previne e trata os distúrbios cinéticos funcionais intercorrentes em órgãos e sistemas do corpo humano ${ }^{10}$.

A atenção básica, segundo o modelo adotado pelo Sistema Único de Saúde (SUS), é definida como a porta de entrada, o contato primário e preferencial da população com os profissionais da saúde. É guiada pelos princípios defendidos pelo SUS universalidade, integralidade e equidade, enfatiza o trabalho em equipe e engloba um conjunto de ações de caráter individual e coletivo, que envolvem promoção da saúde, prevenção de doenças, diagnóstico, tratamento e reabilitação ${ }^{11}$.

Em decorrência das grandes transformações demográficas e epidemiológicas, e com a implantação do PSF atualmente com status de ESF, percebeu-se a necessidade e a importância da inserção do fisioterapeuta na equipe, tendo um enfoque maior na prevenção de doenças, promoção e manutenção da saúde, melhorando, assim, a qualidade de vida da comunidade ${ }^{11}$.

Dessa forma a Estratégia de Saúde da Família (ESF), a partir da sua implantação, produziu mudanças importantes na práxis dos profissionais, levando consequentemente a sua ampliação em todo o sistema de saúde, o Núcleo de Apoio de Saúde da Família (NASF) surge como uma ferramenta que possibilita ressignificações nas práticas dos profissionais que participam nessa política, de toda a equipe trabalhadora da atenção básica, da população assistida e na rede de atenção ${ }^{12}$.

O desafio principal dos profissionais que atuam no NASF é o de desenvolver um novo pensamento de trabalho que utilize a atuação conjunta, integrada e intersetorial, com base nas redes entre os trabalhadores e incorporando a participação dos usuários, refletindo o conceito ampliado de saúde assumido pelo (SUS) ${ }^{12}$. Diante desse contexto, a aproximação entre a fisioterapia e o nível primário apresenta-se como alternativa capaz de tonificar a atenção básica, aumentando a resolutividade do sistema e contribuindo para a garantia da integralidade na assistência ${ }^{13}$.

Assim o fisioterapeuta insere-se no sistema de atenção básica numa nova perspectiva de atuação, concentrado nas práticas de prevenção e promoção e não restrita aos procedimentos de reabilitação, ao 
contrário do que tem predominado nas ações deste segmento profissional ${ }^{7-13}$.

Logo, o presente estudo teve como objetivo geral analisar a atuação do fisioterapeuta no núcleo ampliado a saúde da família e atenção básica (NASF$\mathrm{AB})$ no município de Lucena- PB. E para o determinado estudo foi formulada a seguinte hipótese: As atribuições desenvolvidas pelos fisioterapeutas estão relacionadas à criação de ações de acompanhamento voltadas a pessoa com deficiência, ações de pesquisa específicas de saúde mental, realizações de ações multiprofissionais e dar suporte a ESF.

\section{MATERIAL E MÉTODO}

Essa pesquisa foi caracterizada por ser um estudo de campo, na qual é definido por ter um maior alcance, nas ciências biomédicas, o estudo de campo costuma ser utilizado tanto como estudo principal para esclarecimento do campo da pesquisa em seus múltiplos aspectos quanto para a descrição de síndromes raras. Seus resultados, de modo geral, são apresentados em aberto, ou seja, na condição de hipóteses, não de conclusões ${ }^{14}$.

Teve uma abordagem qualitativa $\mathrm{e}$ quantitativa, no qual a pesquisa qualitativa permite a extração das experiências humanas em relação a um fenômeno estudado na investigação, enquanto que a quantitativa utiliza dados estatísticos para comprovação dos resultados obtidos em pesquisa. A abordagem quanti-qualitativa requer o entendimento sobre o fenômeno de escolha de forma que não se obteria com a utilização de apenas uma abordagem, permitindo a complementação entre palavras e números $^{14}$. O estudo descritivo tem como objetivo principal a descrição das características de determinada população ou fenômeno, e o estudo retrospectivo é construído com base em registros do passado com prosseguimento até o presente e só se torna viável realizá-lo quando há arquivos com protocolos completos e organizados ${ }^{14}$.

$\mathrm{O}$ presente trabalho foi desenvolvido na cidade de Lucena-PB, no qual possui um NASF-AB, serão estudados fisioterapeutas, onde estes são direcionados para 06 Equipes de Saúde da Família (Eq SF) no período de Agosto a Outubro de 2018. A pesquisa foi composta por 5 fisioterapeutas recrutados por conveniência. Como critérios de inclusão foram estabelecidos: atuar no NASF-AB por no mínimo seis meses, atuar no município de Lucena, consentir a participação via assinatura do TCLE.

Foram excluídos desta pesquisa aqueles que se recusarem a responder os questionários, óbito e questionários incompletos. $\mathrm{O}$ procedimento de coleta de dados foi dividido em dois eixos, para melhor organização das atividades a serem executadas: ETAPA I: Antes de iniciar a coleta do material prático, foi realizado o primeiro contato com os sujeitos do estudo, a fim de marcar dia, horário e local que melhor se adequasse aos mesmos, sendo escolhido o local de trabalho de cada participante para realização da entrevista. Após confirmação do aceite, os sujeitos da pesquisa foram informados sobre o objetivo do estudo, contando para isso, com a colaboração da Secretaria Municipal de Saúde da cidade de Lucena-PB. ETAPA II: Consistem na aplicação das entrevistas de acordo com local, data e horários preestabelecidos previamente.

Para a análise e o tratamento do material prático, será utilizada a técnica de análise de conteúdo da comunicação visando obter, por procedimentos sistemáticos e objetivos de descrição do conteúdo das mensagens ${ }^{15}$. A análise temática se desdobrará em três etapas: uma pré-análise em seguida a exploração do material colhido e por último tratamento dos resultados obtidos e interpretação do mesmo. Serão selecionados depoimentos sobre temas/conteúdos convergentes e/ou divergentes para a construção das categorias de análise do material prático $^{15}$.

Estudo de séries de casos é caracterizado por ser um estudo transversal no qual descreve a experiência de um grupo de participantes com diagnósticos semelhantes ${ }^{16}$. Os dados distribuídos foram expostos em tabelas, uma relacionada aos dados sociodemográficos iniciais e outra embasa toda a parte sociodemográfica da relação dos profissionais com o NASF-AB do município.

No Brasil, a pesquisa que envolvem seres humanos devem corresponder a Resolução do Conselho Nacional de Saúde (CNS) no 466/2012 a qual esclarece que, para a realização da pesquisa cientifica envolvendo seres humanos, é necessário visar os princípios de autonomia, não maleficência, beneficência e justiça ${ }^{17}$. Desse modo, antes de iniciar a entrevista, foi apresentado aos participantes do estudo o Termo de Consentimento Livre e Esclarecido, que além de esclarecê-los quanto aos objetivos do estudo e seus possíveis riscos e benefícios, solicitou a autorização dos mesmos para a publicação dos resultados obtidos. O presente estudo foi aprovado pelo Comitê de Ética e Pesquisa (CEP) do Instituto Federal de Ciência e Tecnologia da Paraíba (IFPB) através do parecer 2.906.079.

Através do presente estudo, observam-se benefícios tanto na atualização dos profissionais sobre o papel do fisioterapeuta do NASF-AB como na investigação das atividades desenvolvidas pelo mesmo em seu local de trabalho, contribuindo assim para o avanço da fisioterapia por meio do incentivo a investigação e a execução de novas pesquisas sobre a temática.

\section{RESULTADOS E DISCUSSÃO}

De acordo com os dados da Tabela 1, os fisioterapeutas entrevistados responderam sobre seus 
dados pessoais como idade, sexo, se possuíam pósgraduação, tipo de contratação e ano de conclusão da graduação.

Tabela 1. Respostas referentes ao perfil sociodemográfico dos fisioterapeutas do NASF-AB da cidade Lucena-PB, 2018.

\begin{tabular}{c|c|c|c|c|c}
\hline Fisioterapeuta & Idade & Sexo & Pós-Graduação & $\begin{array}{c}\text { Tipo de } \\
\text { contratação }\end{array}$ & $\begin{array}{c}\text { Ano de } \\
\text { Conclusão da } \\
\text { Graduação }\end{array}$ \\
\hline 1 & 27 & $\mathrm{~F}$ & $\begin{array}{c}\text { Traumato- } \\
\text { Ortopedia }\end{array}$ & $\begin{array}{c}\text { Prestador de } \\
\text { Serviço }\end{array}$ & 2014 \\
\hline 2 & 35 & $\mathrm{~F}$ & $\begin{array}{c}\text { Dermatofunciona } \\
\text { l }\end{array}$ & $\begin{array}{c}\text { Prestador de } \\
\text { Serviço }\end{array}$ & 2008 \\
\hline 3 & 36 & $\mathrm{M}$ & Geriatria & $\begin{array}{c}\text { Prestador de } \\
\text { Serviço }\end{array}$ & 2008 \\
\hline 4 & 37 & $\mathrm{~F}$ & $\begin{array}{c}\text { Traumato- } \\
\text { Ortopedia }\end{array}$ & $\begin{array}{c}\text { Prestador de } \\
\text { Serviço }\end{array}$ & 2013 \\
\hline 5 & 48 & $\mathrm{~F}$ & $\begin{array}{c}\text { Traumato- } \\
\text { Ortopedia, UTI }\end{array}$ & Estatutário & 2014 \\
\hline \multicolumn{2}{|c|}{ Fonte: Dados da Pesquisa, 2018.} & \multicolumn{4}{l}{}
\end{tabular}

De acordo com o questionário realizado cerca de $80 \%$ dos profissionais são compostos de mulheres e $20 \%$ de homens, $100 \%$ dos profissionais são formados após o ano de 2007, 80\% recebem $\mathrm{R} \$$ $1.200,00$ e apenas $20 \%$ ganham R $\$ 1,500,00$ e $100 \%$ deles possuem pós- graduação, $80 \%$ dos instrumentos da coleta são prestadores de serviço e $20 \%$ estatutários.

De acordo com os dados da Tabela 2, os fisioterapeutas entrevistados responderam sobre dados relacionados ao NASF-AB no município, como carga horária semanal, renda mensal, tempo de prática profissional e atividades exercidas no NASF$\mathrm{AB}$.

Tabela 2. Dados sociodemográficos referente ao NASF-AB da cidade de Lucena, 2018

\begin{tabular}{c|c|c|c|c}
\hline Fisioterapeuta & $\begin{array}{c}\text { Carga Horária } \\
\text { Semanal do } \\
\text { NASF-AB }\end{array}$ & $\begin{array}{c}\text { Renda Mensal } \\
\text { no NASF-AB }\end{array}$ & $\begin{array}{c}\text { Tempo de } \\
\text { Prática } \\
\text { Profissional no } \\
\text { NASF-AB }\end{array}$ & $\begin{array}{c}\text { Atividade Exercida no } \\
\text { NASF-AB }\end{array}$ \\
\hline 1 & $20 h$ & 1,500 & 3 anos & $\begin{array}{c}\text { Domiciliar } \\
\text { Em grupo }\end{array}$ \\
\hline 2 & $20 h$ & 1,200 & 3 anos & $\begin{array}{c}\text { Domiciliar } \\
\text { Em Grupo Educativas } \\
\text { em Equipe } \\
\text { Assistência e } \\
\text { Atividades de Atenção } \\
\text { a Saúde, Identificação } \\
\text { de Demandas, } \\
\text { Acolhimento e } \\
\text { Assistência }\end{array}$ \\
\hline 3 & $20 h$ & 1,200 & 5 anos & $\begin{array}{c}\text { Ambulatorial } \\
\text { Domiciliar }\end{array}$ \\
\hline 4 & $20 h$ & 1,200 & 3 anos & $\begin{array}{c}\text { Domiciliar } \\
\text { Em Grupo, } \\
\text { Educativas e em } \\
\text { Equipe } \\
\text { Assistência e }\end{array}$ \\
\hline 5 & $30 h$ & 1,200 & 3 anos & $\begin{array}{c}\text { Atividades de Atenção } \\
\text { a Saúde, Identificação } \\
\text { de Demandas }\end{array}$ \\
\hline Fonte: Dados da Pesquisa, 2018. & & &
\end{tabular}

Em relação à Tabela supracitada observa-se que $80 \%$ dos profissionais cumprem a carga horária mínima exigida pelo NASF-AB de 20 horas semanais, e $20 \% 30$ horas sendo esta a carga horária máxima exigida, $80 \%$ da amostra trabalham em outro local fora o NASF- AB e $20 \%$ não, $60 \%$ exercem atividades como atendimento domiciliar e ambulatorial, atendimentos em grupos, atividades educativas em equipe, assistência e atividade de atenção em saúde para a população, identificação de demanda, acolhimento e assistência, 20\% dos instrumentos utilizados só realizam apenas atividades ambulatoriais e domiciliar, dos 05 profissionais estudados $100 \%$ tem mais de 3 anos de prática profissional no NASF-AB do município de LucenaPB.

Segundo o banco de dados online do DAB/SAS/MS a cidade de Lucena-PB é composta por mais de 12 mil habitantes no qual 04 equipes de saúde da família começaram a ser inseridas no ano de 2001 até o ano de 2004, no ano de 2005 o número diminuiu onde 03 equipes cobriam toda a área, a partir do ano de 2006 até 2013, a cidade continha 05 equipes e no ano de 2014 até o momento de hoje 07 equipes tem feito esse papel. A distribuição das equipes de saúde da família (ESF) na cidade de Lucena está à cima de $81 \%$. Em relação ao NASF$\mathrm{AB}$ na cidade de Lucena-PB, teve seu início no ano de 2013 até os dias de hoje, no qual 01 NASF-AB faz a cobertura de toda a cidade. O NASF-AB da cidade de Lucena está inserido na modalidade de NASF-AB 1 , devendo estar vinculada a no mínimo 05 (cinco) e no máximo 09 (nove) equipes de saúde da família (Eq SF) e equipes de Atenção Básica.

Os fisioterapeutas entrevistados responderam qual seria a sua análise sobre o NASF-AB no município, quais atividades e projetos desenvolvidos e se eles consideravam essas atividades atribuições do fisioterapeuta do NASF- AB. E foi identificado que o NASF-AB no município tem tido uma cobertura muito boa, completa e diferenciada por ser um lugar que não oferece recursos, oferece uma acessibilidade muito boa $^{18}$. identificando que o profissional que atua no NASF-AB tem como princípios básicos em sua atividade a integralidade, $\mathrm{o}$ conhecimento de território, a humanização, a educação popular e permanente em saúde, a interdisciplinaridade e a intersetorialidade, direcionados para ações de promoção de saúde que interfiram diretamente na qualidade de vida dos cidadãos ${ }^{18}$.

Em relação às atividades e projetos desenvolvidos pelo Fisioterapeuta no NASF-AB, foram identificados a realização de várias atividades, cada um na sua área porém todos trabalhando em conjunto com foco na parte infanto-juvenil, palestras nas escolas e uma atenção especial com o idoso, no qual foi exposto que o fisioterapeuta pode atuar com aulas em grupo de atividades físicas específicas para idosos $^{18}$.

$\mathrm{O}$ atendimento de fisioterapia passou de uma visão de atenção às incapacidades instaladas que prejudicassem o movimento para uma visão focada em considerar as características do movimento como um contínuo entre condições sadias e patológicas e não mais situações dicotômicas, estando em uma ou outra $^{18}$. Sobre essas atividades serem atribuições do fisioterapeuta do NASF-AB, $60 \%$ dos entrevistados 
mostram que sim, e $40 \%$ identificaram que não, não estando de acordo com estudos na area $^{3}$, no qual ele mostra que o fisioterapeuta está apto a planejar, implementar, e executar a política, programas, recursos, pesquisas ou eventos em saúde pública, contribuindo para o planejamento, a investigação e estudos epidemiológicos da população ${ }^{3}$.

De acordo com os dados da Tabela 3, os fisioterapeutas entrevistados responderam como se estabelecia a relação entre o fisioterapeuta e os demais profissionais, qual seria as principais demandas e como se estabelece a relação entre o fisioterapeuta e o usuário.

Tabela 3. Entrevista para levantamento de dados sobre as atribuições do fisioterapeuta do NASF-AB no município de Lucena-PB, 2018

\begin{tabular}{|c|c|c|c|}
\hline Fisioterapeuta & $\begin{array}{l}\text { Como se estabelece a relação } \\
\text { entre o Fisioterapeuta do } \\
\text { NASF- AB e os demais } \\
\text { profissionais? }\end{array}$ & \begin{tabular}{|c|} 
Quais as \\
principais \\
demandas dos \\
usuários postas \\
ao \\
Fisioterapeuta \\
NASF-AB? \\
\end{tabular} & $\begin{array}{l}\text { Como se estabelece a } \\
\text { relação entre o } \\
\text { fisioterapeuta e } \\
\text { usuário? }\end{array}$ \\
\hline 1 & $\begin{array}{c}\text { Éuma interação } \\
\text { multidisciplinar porque um } \\
\text { sempre vai precisar do outro. } \\
\text { Eu tenho um paciente acamado } \\
\text { e vejo que o mesmo precisa de } \\
\text { uma nutricionista, eu vou e } \\
\text { peço encaminhamento para ela } \\
\text { vir atender meu paciente". }\end{array}$ & $\begin{array}{c}\text { "Principalmente } \\
\text { pessoas com } \\
\text { seqüelas de AVE, } \\
\text { fraturas e pós } \\
\text { cirurgias". }\end{array}$ & $\begin{array}{l}\text { "Bem aberta, estamos } \\
\text { sempre nos PSF } \\
\text { perguntando sobre } \\
\text { algum } \\
\text { encaminhamento" }\end{array}$ \\
\hline 2 & $\begin{array}{l}\text { "A gente consegue ter uma } \\
\text { interação bem bacana, sempre } \\
\text { quando um está precisando a } \\
\text { gente está encaminhando, em } \\
\text { relação das atividades a gente } \\
\text { sempre está entrando em } \\
\text { comunicação, procurando } \\
\text { sempre trabalhar em grupo. Eu } \\
\text { acho que o intuito do NASF-AB } \\
\text { é isso, todos os } \\
\text { profissionais trabalhando em } \\
\text { grupo". }\end{array}$ & $\begin{array}{l}\text { "No município a } \\
\text { gente tem muito } \\
\text { paciente com } \\
\text { hipertensão, que } \\
\text { é um grande } \\
\text { fator de risco } \\
\text { para o AVC, } \\
\text { paciente } \\
\text { debilitado, } \\
\text { diabético } \\
\text { idoso,diabético } \\
\text { muitos acidentes } \\
\text { de moto, } \\
\text { deformidade, } \\
\text { pós- operatório } \\
\text { imediato". }\end{array}$ & $\begin{array}{l} \\
\text { "E uma relação bem } \\
\text { tem medo de dar o } \\
\text { telefone ao paciente, até } \\
\text { por uma particularidade } \\
\text { dele não pode vir no dia, } \\
\text { a gente sempre está } \\
\text { entrando em contato". }\end{array}$ \\
\hline 3 & $\begin{array}{l}\text { "Muito boa, nós sempre } \\
\text { trabalhos em grupo, o } \\
\text { fisioterapeuta com o Educador } \\
\text { Físico, um apoiando um outro, } \\
\text { indicando e encaminhando } \\
\text { pacientes, procurando sempre } \\
\text { trabalhar em grupo". }\end{array}$ & \begin{tabular}{|} 
"A Maior \\
demanda é \\
acidente \\
automobilístico, \\
AVC e \\
Problemas"
\end{tabular} & $\begin{array}{l}\text { "A aceitação do } \\
\text { tratamento é muito boa, } \\
\text { e alguns a gente até } \\
\text { consegue ter um vínculo } \\
\text { maior, amigos fora do } \\
\text { ambiente de trabalho". }\end{array}$ \\
\hline 4 & "Bоа" & $\begin{array}{l}\text { "Geralmente } \\
\text { artrose" }\end{array}$ & $\begin{array}{l}\text { "A minha relação é } \\
\text { ótima, nunca tive } \\
\text { problema com } \\
\text { ninguém". }\end{array}$ \\
\hline 5 & $\begin{array}{l}\text { Pelo menos aqui temos uma } \\
\text { boa relação, se um paciente } \\
\text { precisou de um nutricionista a } \\
\text { gente consegue psicólogo da } \\
\text { mesma forma". }\end{array}$ & $\begin{array}{c}\text { "Pessoas com } \\
\text { Artrose, Artrite e } \\
\text { atendemos } \\
\text { muito traumato } \\
\text { aqui". }\end{array}$ & $\begin{array}{c}\text { "Da minha parte é } \\
\text { muito boa, me relaciono } \\
\text { muito bem com meus } \\
\text { pacientes". }\end{array}$ \\
\hline
\end{tabular}

Fonte: Dados da Pesquisa, 2018.

De acordo com a Tabela 3, em relação aos fisioterapeutas e os demais profissionais, os instrumentos da pesquisa mostram que existe uma relação multidisciplinar, e que essa relação é dita como muito boa, porém não corrobora com um estudo semelhante ${ }^{13}$, no qual ele diz que o fisioterapeuta deve ter uma atenção maior com outros campos da área de saúde, torna-se necessária uma aproximação da fisioterapia com a vigilância sanitária, vigilância epidemiológica, vigilância nutricional, vigilância à saúde do trabalhador e vigilância ambiental, com vistas à identificação e acompanhamento de problemas que requerem atenção contínua, ação sobre territórios definidos e articulação entre as ações promocionais, preventivas, curativas e reabilitadoras $^{13}$.
Sobre a demanda, os profissionais citam que existe uma particularidade quando se fala em pessoas com hipertensão arterial, AVC, problemas de coluna e acidentes automobilísticos, não estando de acordo com estudos ${ }^{13-19}$, mostrando que uma das principais dificuldades enfrentadas para desempenho das atividades de competência da fisioterapia no NASF foi a falta de conhecimento de profissionais da saúde e usuários em relação ao papel do fisioterapeuta na Atenção Básica. Muitos profissionais acreditavam que as atividades não eram necessárias e que o trabalho do fisioterapeuta deveria ser focar nos atendimentos de pacientes acamados com disfunções respiratórias, traumatológicas, neurológicas e dentre outras, ou seja, o exercício de conduta puramente curativa e reabilitadora ${ }^{19}$. A relação entre o fisioterapeuta e o usuário mostrou-se muito boa, no qual os profissionais citam uma aproximação muito grande com seus pacientes. Os fisioterapeutas entrevistados responderam sobre estratégias de mobilização social se existe espaço de qualificação profissional e quais os principais desafios postos ao fisioterapeuta do NASF-AB. As estratégias de mobilização social e participação democrática os fisioterapeutas realizam atividades como palestras e toda a parte de educação em saúde, possibilitando que a população conheça mais sobre o papel do fisioterapeuta concordando com um estudo, que informou que atenção básica de saúde é um anexo de intervenções em saúde no âmbito individual e coletivo que envolve: promoção, prevenção, diagnóstico, tratamento e reabilitação com ações democráticas e participativas ${ }^{20}$.

Em relação ao espaço de qualificação profissional, na cidade de Lucena-PB ainda não existe esse tipo de local, mas os profissionais relataram que a gestão sugere algo como um suporte20.

Os desafios postos ao fisioterapeuta do NASF-AB foi identificado que a falta de equipamentos ainda é um grande problema para os profissionais, o meio de locomoção para chegar ao paciente também foi bastante citado, tornando a evolução do paciente um pouco mais lenta, corroborando com estudos ${ }^{2-20}$, no qual é dito que o redirecionamento está explicita a uma concepção de trabalho em saúde baseada nos desafios ético-político da transdisciplinaridade, multiprofissionalidade e integralidade, que habitam o campo paradigmático da reorientação das práticas de atenção do SUS e de sua Estratégia de Saúde da Família (ESF) ${ }^{20}$

Os fisioterapeutas entrevistados responderam se tem sido realizada ações multiprofissionais e atividades de promoção e proteção à saúde, se foram criados acompanhamentos a pessoa com deficiência e se tem sido pesquisa relacionadas a saúde mental. As ações multiprofissionais têm sido realizadas em conjunto com toda equipe, no qual foi mostrado que 
os profissionais realizam palestras sobre tabagismos, saúde da mulher, saúde do homem ${ }^{21}$, mostrando que as ações multiprofissionais são atividades novas inseridas na comunidade onde a ESF atua em uma vertente com um foco maior na assistência, com marcações e realizações de consultas e encaminhamentos para exames complementares ${ }^{21}$.

A atuação no NASF-AB propõe visitas para acompanhamento do usuário e orientação aos cuidadores e ACS no processo de reabilitação, proporcionando uma atenção onde existe a responsabilidade dos envolvidos no cuidado e não uma dependência do usuário à presença do fisioterapeuta. As pesquisas em conjunto a equipe sobre a saúde mental não têm sido realizadas por nenhum dos profissionais ${ }^{4-20}$.

\section{CONCLUSÃO}

A quantidade de fisioterapeutas atuantes no NASF-AB da cidade Lucena-PB é relativamente proporcional ao seu número de habitantes. Os fisioterapeutas que atuam no NASF-AB 1 da referida cidade paraibana têm desenvolvido ações de saúde como atividades domiciliares e ambulatoriais, atividades em grupo, atividades educativas em equipe, assistência e atividades de atenção em saúde para a população, acolhimento e assistência.

Com relação a amostra entrevistada, a relação entre o fisioterapeuta e os demais profissionais tem sido satisfatória, mostrando à importância do atendimento multiprofissional no qual tem sido realizadas palestras em conjunto bem como encaminhamentos de pacientes para as diversas áreas, a relação entre o fisioterapeuta e o paciente tem se mostrado excelente no qual os profissionais tem um contato muito próximo com o mesmo, identificando assim a realização das atribuições cabidas ao fisioterapeuta no município.

Salienta-se, por fim, a necessidade de se verificar com maior profundidade como o processo de trabalho dos fisioterapeutas que atuam no NASF$\mathrm{AB}$ pode contribuir para transformar a prática profissional no nível de Atenção Básica, ampliando suas competências e habilidades na atuação interdisciplinar e desenvolvimento de ações de prevenção de doenças e promoção à saúde nos territórios.

\section{REFERÊNCIAS}

1. David MLO, Ribeiro MAGO, Zanolli ML, Mendes RT, Assumção MS, Schinviski CIS. Proposta de atuação da fisioterapia na saúde da criança e do adolescente: uma necessidade na atenção básica. Saúde debate. 2013;37(96):120-29.

2. Neves LMT, Aciole GG. Desafios da integralidade: revisitando as concepções sobre o papel do fisioterapeuta na equipe de Saúde da Família. Interface (Botucatu). 2011;15(37):551-64.
3. Barbosa EG, Ferreira DLS, Furbino, SAR. Experiência da fisioterapia no Núcleo de Apoio à Saúde da Família em Governador Valadares, MG. Fisioter mov. 2010;23(2):86-95.

4. Formiga NFB, Ribeiro KSQS. Inserção do fisioterapeuta na Atenção Básica: uma Analogia entre Experiências Acadêmicas e a Proposta dos Núcleos de Apoio à Saúde da Família (NASF). Rev bras ciênc saúde, 2012;16(2):113-22.

5. Ribeiro CD, Flores-Soares MC. Desafios para a inserção do fisioterapeuta na atenção básica: o olhar dos gestores. Rev Salud Pública, 2015;17(3):379-93.

6. Rodrigues F, Bitencourt LTG. Atuação da Fisioterapia no Programa de Residência Multiprofissional. Rev Programa de Residência Multiprofissional em Atenção Básica/Saúde da Família. 2013;1:42-52.

7. Aveiro MC, Aciole GG, Driusso P, Oish J. Perspectivas da participação do fisioterapeuta no Programa Saúde da Família na atenção à saúde do idoso. Ciênc saúde coletiva. 2011; 16(Suppl 1):1467-78.

8. Souza MC, Araújo TM, Reis Júnior WM, Souza JN, Vilela ABA, Franco TB. Integralidade na atenção à saúde: um olhar da equipe de saúde da família sobre a fisioterapia. Mundo saúde. 2012;36(3):452-60.

9. Augusto VG, Aquino CF, Machado NC, Cardoso VA, Ribeiro S. Promoção de saúde em unidades básicas: análise das representações sociais dos usuários sobre a atuação da fisioterapia. Ciênc saúde coletiva. 2011;16(Suppl 1):957-63.

10. Carvalho STRF, Caccia-Bava, MCGG. Conhecimentos dos usuários da Estratégia Saúde da Família sobre a fisioterapia. Fisioter mov. 2017;24(4):655-64.

11. Loures LF, Silva MCS. A interface entre o trabalho do agente comunitário de saúde e do fisioterapeuta na atenção básica à saúde. Ciênc saúde coletiva. 2010;15(4):2155-64.

12. Bispo Júnior JP. Fisioterapia e saúde coletiva: desafios e novas responsabilidades profissionais. Ciênc saúde coletiva. 2010;15(Suppl 1):1627-36.

13. Maciel MS, Coelho MO, Marques LARV, Rodrigues Neto EM, Lotif MAL, Ponte ED. Ações de saúde desenvolvidas pelo núcleo de apoio à saúde da família-NASF. Saúde (Santa Maria). 2015;41(1):117-22.

14. Oliveira AKP, Borges DF. Programa de Saúde da Família: uma avaliação de efetividade com base na percepção de usuários. Rev Adm Pública. 2008;42(2):369-89.

15. Oliveira G, Andrade ES, Santos ML, Matos GSR. Conhecimento da equipe de saúde da família acerca da atuação do fisioterapeuta na atenção básica. Rev Bras Promoç Saúde (Fortaleza). 2011;24(4):332-39. 
16. Salinero JG. Estudos descriptivos. Nure Investigación. 2004;7.

17. Fernandes TL, Nascimento CMB, Sousa FOS. Análise das atribuições dos fonoaudiólogos do NASF em municípios da região metropolitana do Recife. Rev CEFAC. 2013;15(1):153-59.

18. Souza FLD. Implantação do Núcleo de Apoio à Saúde da Família: percepção do usuário. Saúde debate. 2013;37(97):233-40.

19. Souza MC, Bomfim AS, Souza JN, Franco TB. Fisioterapia e Núcleo de Apoio à Saúde da Família: conhecimento, ferramentas e desafios. Mundo saúde; 2013;37(2):176-84.

20. Castro PC. Papel do fisioterapeuta na universidade aberta da terceira idade de São Carlos. Estud interdiscipl envelhec. 2014; 19(1):287-305.

21. Dias RC. Atenção básica à saúde do idoso no Brasil: limitações e desafios. Geriatria \& Gerontologia. 2008;3(2):122-25

\section{CONFLITO DE INTERESSES}

Os autores declaram não haver conflitos de interesse.

\section{AUTOR PARA CORRESPONDÊNCIA}

\section{Weslley Barbosa Sales}

weslleyssales8@gmail.com

Submetido em 01/03/2020

Aceito em 20/07/2020 